\title{
Color, contenido de antocianinas y dimensiones de semilla en líneas endogámicas de maíz azul y sus cruzas
}

\author{
Color, anthocyanin content and seed dimensions in \\ blue maize inbred lines and their crosses \\ José Luis Arellano Vázquez $z^{1}$, Alejandro Herrera Zamora², \\ Germán Fernando Gutiérrez Hernández ${ }^{2 *}$, Luis Fernando Ceja Torres ${ }^{3}$, Estela Flores Gómez ${ }^{2}$
}

\section{RESUMEN}

El grano de maíz azul contiene antocianinas que le confieren su color exótico y es apreciado en la cocina mexicana tradicional. Actualmente se considera un alimento funcional por sus aportaciones a la salud humana pero su producción es insuficiente para abastecer el mercado. Una opción para incrementar su disponibilidad es desarrollar híbridos con rendimiento y contenido del pigmento elevados. El objetivo de este trabajo fue examinar las relaciones entre las dimensiones, las coordenadas del color y el contenido de antocianinas en semillas de líneas parentales de maíz azul y en sus cruzas simple y trilineal. Se utilizaron semillas del híbrido trilineal experimental de maíz azul GIIM AZUL 10E y de sus progenitores (CEVAMEX - INIFAP, México). Se empleó un diseño completamente al azar con cuatro repeticiones ( 25 semillas). Se evaluaron las dimensiones de las semillas, el contenido de antocianinas y las coordenadas del color ( $\mathrm{L}^{*}, \mathrm{a}^{*}, \mathrm{~b}^{*}, \mathrm{c}$ y h). Los resultados se sometieron a análisis de varianza, comparación de medias (Tukey, $\mathrm{P} \leq 0,05$ ), pruebas de correlación lineal y de regresión múltiple. Los experimentos se realizaron en la UPIBI - IPN (Ciudad de México). Se observó que las semillas de las líneas parentales, en comparación con las cruzas, mostraron dimensiones menores, su color azul fue más luminoso, de menor pureza (grisáceo) y con saturación escasa (poco vívido), y su contenido de antocianinas resultó mayor. Los valores de correlación y de regresión indicaron que el contenido de antocianinas se asoció inversamente con las dimensiones de las semillas y, directamente, con el color azul poco saturado y de luminosidad reducida. Las variables con mayor contribución a la cantidad de antocianinas fueron, negativamente, el peso de semilla y la saturación, y, de manera positiva, la pureza del color.

Palabras clave: Zea mays L., antioxidantes, maíces pigmentados, morfología de semillas, pigmentos vegetales.

\section{ABSTRACT}

The blue corn grain contains anthocyanins that give it its exotic color, it is appreciated in traditional Mexican gastronomy, it is currently considered a functional food for its contributions to human health, and its production is insufficient to supply the market. One option to increase its availability is to develop hybrids with high yield and pigment content. The objective of the present work was to examine the relationships between dimensions, color coordinates, and anthocyanin content in seeds of blue corn parental lines and their simple and trilinear crosses. Seeds of the experimental trilinear blue maize hybrid GIIM AZUL $10 E$ and its parents (CEVAMEX - INIFAP, Mexico) were used. A completely randomized design with four replications (25 seeds) was used. Seed dimensions, anthocyanin content, and color coordinates $\left(L^{*}, a^{*}, b^{*}, c, h\right)$ were evaluated. The results were subjected to analysis of variance, means comparison (Tukey, $P \leq 0.05$ ), linear correlation test, and multiple regression test. The experiments were carried out at UPIBI - IPN (Mexico City). It was obtained that the seeds of the parental lines, in comparison with the crosses, showed smaller dimensions, their blue color was more luminous, of lower purity (grayish) and with scarce saturation (little vivid) and their anthocyanin content was higher. Correlation and regression values indicated that anthocyanin content was inversely associated with seed dimensions and directly with low saturated blue color and reduced luminosity. The variables with the greatest contribution to the level of anthocyanins were, negatively, seed weight and saturation, and, positively, color purity.

Keywords: Zea mays L., pigmented corn, seed morphology, plant pigments, antioxidants.

1 Instituto Nacional de Investigaciones Forestales, Agrícolas y Pecuarias. Campo Experimental Valle de México. C.P. 56250. Coatlinchán, estado de México, México.

2 Instituto Politécnico Nacional. Unidad Profesional Interdisciplinaria de Biotecnología. Av. Acueducto s/n. La Laguna Ticomán. C.P. 07340. Ciudad de México, México.

3 Instituto Politécnico Nacional. Centro Interdisciplinario de Investigación para el Desarrollo Integral Regional Unidad Michoacán. Justo Sierra 28. C.P. 59510. Jiquilpan, Michoacán, México.

* Autor para correspondencia: gfgutierrez@ipn.mx.

Fecha de Recepción: 21 de Marzo, 2021.

Fecha de Aceptación: 6 de Junio, 2021. 


\section{Introducción}

La variabilidad genética del maíz en México es vasta (Kato et al., 2009) y es esencial conocerla y aprovecharla, entre otras finalidades, para generar maíces mejorados con valor agregado como los pigmentados (azules, morados, rojos y rosados), en los cuales el pigmento se sintetiza en la semilla (Yang et al., 2009) y son la base de un sinnúmero de platillos típicos.

El color de la semilla del maíz azul se debe a las antocianinas (Salinas et al., 2013), pigmentos vegetales derivados de la cianidina (Salinas et al., 1999). Las antocianinas se localizan en el pericarpio y/o en la capa de aleurona (Betrán et al., 2001; Hernández-Uribe et al., 2007). Son solubles en agua y actúan como antiinflamatorias y antioxidantes, reducen el colesterol y triglicéridos en la sangre, inhiben la peroxidación de lípidos en el hígado y se relacionan con la reducción de cáncer (Wang y Zeng, 2009; Soto-Vaca et al., 2012). Por otra parte, las antocianinas del maíz se utilizan en la elaboración de colorantes de vinos, mermeladas, jugos de fruta y textiles (Boo et al., 2012).

El exotismo de los maíces pigmentados reside esencialmente en su color llamativo. Cada una de sus variantes posee graduaciones de color específicas. Pese a su importancia, la cuantificación de antocianinas solo se ha hecho en algunas razas de maíz (López-Martínez et al., 2009) y la descripción cuantitativa del color de la semilla del maíz azul también es escasa.

Los parámetros o coordenadas del color $\left(L^{*}, a^{*}, b^{*}\right)$ fueron establecidos por la Comisión Internacional de Iluminación (CIE, 2004). L* es la luminosidad del color $(0=$ oscuro a $100=$ blanco $) ; a^{*}$ alude a los colores morado a rojo (valores positivos) o morado a verde (valores negativos); $b^{*}$ se refiere a los colores morado hacia amarillo (valores positivos) o morado hacia azul (valores negativos). A partir de $a^{*}$ y b* se calculan las coordenadas $\mathrm{c}$ y h que corresponden, respectivamente, al color (croma o matiz) particular, que va del gris al color puro; y al grado de saturación del color, cuya tonalidad a $0^{\circ}$ es roja, a $90^{\circ}$ amarilla, a $180^{\circ}$ verde y a $270^{\circ}$ azul (McGuire, 1992).

En el Altiplano Central de México (estados de México, Hidalgo, Puebla y Tlaxcala, con 2400 - 2800 msnm) se siembran 150 mil hectáreas con maíz azul que producen 300 mil toneladas de grano destinadas a la elaboración de alimentos tradicionales: tortillas, tlacoyos, quesadillas, harina para atole y pinole (Arellano et al., 2014); mientras que la demanda de este tipo de maíz en esa región es de 450 mil toneladas para aprovechamiento tradicional y 5 mil para la industria harinera.

Para cubrir esa demanda se requieren nuevas variedades o híbridos que dupliquen el rendimiento actual y que tengan resistencia al acame, textura de grano semicristalina, color del grano azul intenso, tolerancia a plagas y enfermedades, y, además, con estructura de planta adecuada para siembras mecanizadas y con alta densidad de población. Aunado a lo anterior, el precio del maíz azul es de 70 a $80 \%$ mayor que el del blanco, así que su rentabilidad y potencial son amplios (Arellano et al., 2013).

A través del mejoramiento genético convencional es factible elevar los rendimientos unitarios de maíz azul, mediante variedades de polinización libre o híbridos. Con estos últimos se puede aumentar la producción de 50 a $60 \%$ (Lee y Tollenaar, 2007).

Para desarrollar híbridos de maíz azul conviene seleccionar el sexo de las líneas parentales según la ubicación de las antocianinas en la semilla (Cui et al. 2012), ya sea el pericarpio (de origen materno), la aleurona (con origen en la recombinación de ambos progenitores), o bien, en ambas estructuras. Además, las antocianinas asumen su mayor nivel a los 35 días del periodo de llenado de grano y la luz afecta su síntesis.

En el proceso genotécnico es importante conocer la acción génica que controla los caracteres de interés en los progenitores (Gutiérrez et al., 2014). Por ello, se evalúa la aptitud combinatoria de los genotipos sobresalientes y se seleccionan los progenitores con un comportamiento promedio aceptable en los cruzamientos. En el Programa de Maíz del Campo Experimental Valle de México (CEVAMEX) se hizo el mejoramiento genético de maíces azules a partir de criollos sobresalientes colectados en el Altiplano Central de México.

Por la importancia del maíz azul y con la finalidad de coadyuvar a la obtención de híbridos con cualidades agronómicas, tecnológicas y organolépticas que satisfagan la creciente demanda de esta variedad, se efectuó este estudio, cuyo objetivo fue examinar las relaciones entre las dimensiones, coordenadas del color y contenido de antocianinas en semillas de líneas parentales de maíz azul y en sus cruzas simple y trilineal. 
Se considera que el conocimiento de los efectos de los componentes de rendimiento de semilla y de las coordenadas del color sobre la cantidad de antocianinas presentes en semillas completas de líneas parentales de maíz azul, así como el de sus modificaciones al hibridarse, aportará criterios de mejoramiento útiles para la conformación de híbridos de este maíz pigmentado.

\section{Materiales y métodos}

\section{Material genético}

Se utilizaron semillas del híbrido trilineal experimental de maíz azul GIIM AZUL 10E y de los genotipos (Tabla 1) que lo conformaron (líneas parentales y cruzas simple y triple). Este material genético se desarrolló en el Programa de Maíz del Campo Experimental Valle de México del Instituto Nacional de Investigaciones Forestales, Agrícolas y Pecuarias (CEVAMEX, INIFAP).

Los análisis de laboratorio se efectuaron en el 2018 en la Unidad Profesional Interdisciplinaria de Biotecnología del Instituto Politécnico Nacional. Las semillas empleadas en los experimentos se tomaron aleatoriamente de la muestra de trabajo, previamente homogeneizada, que se formó con las semillas de la porción media de la mazorca de 10 plantas con competencia completa de cada genotipo y repetición.

\section{Características morfológicas de las semillas}

Dimensiones. Peso de 100 semillas (P100): se tomaron al azar 100 semillas y se pesaron $(\mathrm{g})$ en balanza analítica (AE Adam P W 184, precisión de $0,1 \mathrm{mg}$ ). La anchura (AG), el espesor (EG) y la longitud (LG) de las semillas se midieron (mm) con vernier digital (Mitutoyo CD-6 CSX). Se calculó el volumen (VOL, $\mathrm{mm}^{3}$ ), el volumen relativo $\left(\mathrm{VR}, \mathrm{g} / \mathrm{cm}^{3}\right)$ y la densidad relativa (DR, $\mathrm{g} / \mathrm{cm}^{3}$ ) de las semillas, con base en las guías de descriptores varietales para maíz del IBPGR (1991) y del SNICS (2014).

\section{Contenido de antocianinas}

Para la cuantificación de antocianinas totales (AT) se molieron 25 semillas completas, al 12\% de humedad, de cada genotipo por repetición, en un molino tipo ciclónico (UDY, 3010-080P, con malla de $0,5 \mathrm{~mm}$ ). La harina se deshidrató en estufa a $65^{\circ} \mathrm{C}$ durante $16 \mathrm{~h}$. Se emplearon $20 \mathrm{mg}$ de la molienda para la determinación de antocianinas mediante el método descrito por Galicia et al. (2012). Los resultados se expresaron, en base seca, con referencia al cloruro de pelargodinina ( $\mu \mathrm{g} \mathrm{Pel} / \mathrm{g}$ ), para lo cual se preparó una curva patrón $(100 \mu \mathrm{g} / \mathrm{mL})$ con esta antocianina.

\section{Coordenadas del color de las semillas (CIE)}

Se digitalizaron las imágenes de las semillas con un escáner y se determinaron los parámetros de color L*, a*, b*, c y h (Alvis et al., 2017) con el software Adobe Photoshop CS3 (Adobe Systems Inc. USA). Se fijaron cuatro puntos de medición en cada lado (ventral y dorsal) de la semilla y se promediaron los valores de las coordenadas para cada semilla y repetición.

\section{Análisis estadístico}

Los experimentos se manejaron según un diseño experimental completamente al azar con cuatro

Tabla 1. Líneas endogámicas y cruzas simple y trilineal de maíz azul bajo estudio (Campo Experimental Valle de México, INIFAP. Chapingo, México, 2017).

\begin{tabular}{cccc}
\hline Tratamiento & Descripción & Autofecundaciones & Origen (2017) \\
\hline \multicolumn{4}{c}{ Líneas endogámicas } \\
\hline 2 & $\mathrm{CH} 1$ & $\mathrm{~S}_{5}$ & Chapingo, México \\
3 & $\mathrm{CH} 2$ & $\mathrm{~S}_{3}$ & Chapingo, México \\
& $\mathrm{CH} 3$ & $\mathrm{~S}_{5}$ & Chapingo, México \\
\hline & & Cruzas & \\
\hline & & & Chapingo, México \\
5 & $\mathrm{CH} 1 \times \mathrm{CH} 2$ & Chapingo, México \\
\hline
\end{tabular}


repeticiones de 25 semillas. La significancia de los tratamientos se probó con un análisis de varianza y sus promedios se compararon mediante la prueba de Tukey $(\mathrm{P} \leq 0,05)$. Se evaluaron las asociaciones entre variables con la prueba de correlación lineal de Pearson $(\mathrm{P} \leq 0,05 ; \mathrm{P} \leq 0,01)$ y se probó el modelo de regresión múltiple AT $=\mathrm{P} 100 \mathrm{~L}^{*} \mathrm{~b} * \mathrm{c}$ h. El procesamiento estadístico de los resultados se hizo con el programa SAS (SAS, 2002).

\section{Resultados y discusión}

Todas las variables resultaron altamente significativas $(\mathrm{P} \leq 0,01)$ en el análisis de varianza (Tabla 2), con coeficientes de variación reducidos, excepto los concernientes a los parámetros del color a* $(40,7)$ y h $(51,7)$. Esto indicó que, dentro de genotipos, las semillas fluctuaron considerablemente en el color de morado hacia rojo $\left(a^{*}\right)$ y en la saturación o vividez (h) de este. En este sentido, Mendoza-Mendoza et al. (2017) reportan coeficientes de variación altos en todos los parámetros del color y los atribuyen a que el color no es uniforme en las semillas de maíz.

El modelo experimental utilizado resultó adecuado porque sus coeficientes de determinación fueron mayores del $80 \%$ y, por tanto, los experimentos fueron robustos. El material genético empleado en el estudio presentó variabilidad alta entre tratamientos (genotipos) y en todas las mediciones, así que se procedió a la comparación de sus valores medios.

Las semillas de ambas cruzas tuvieron valores mayores $(\mathrm{P} \leq 0,05)$ que las líneas en todos los descriptores físicos evaluados (P100, VR, LG, AG, EG, VOL y DR) (Tabla 3), hecho que se puede

Tabla 2. Cuadrados medios y significancia estadística de las variables evaluadas en las líneas endogámicas y cruzas simple y trilineal de maíz azul bajo estudio (Instituto Politécnico Nacional, México, 2018).

\begin{tabular}{lrrrrr}
\hline Variable $(\mathrm{g} 1=4)$ & C.V. $(\%)$ & $\mathrm{R}^{2}(\%)$ & Cuadrado medio & Error & Significancia \\
\hline P100 & 4,2 & 98 & 157,6 & 1,1 & $* *$ \\
VR100 & 4,8 & 96 & 94,4 & 1,0 & $* *$ \\
LG & 3,4 & 89 & 4,4 & 0,2 & $* *$ \\
AG & 3,5 & 87 & 1,6 & 0,1 & $* *$ \\
EG & 4,2 & 72 & 0,4 & 0,04 & $* *$ \\
VOL & 6,6 & 91 & 24534,6 & 650,6 & $* *$ \\
DR & 6,5 & 80 & 0,1 & 0,01 & $* *$ \\
AT & 0,7 & 99 & 114850,7 & 11,7 & $* *$ \\
L* & 7,9 & 68 & 40,6 & 5,0 & $* *$ \\
a* & 40,7 & 81 & 8,6 & 0,5 & $* *$ \\
b* & 1,0 & 89 & 12,3 & 0,4 & $* *$ \\
c & 18,6 & 91 & 8,84 & 0,23 & $* *$ \\
h & 51,7 & 67 & 67728,5 & 8944,8 & $* *$ \\
\hline
\end{tabular}

g 1 = Grados de libertad, C.V. = Coeficiente de variación, $\mathrm{R}^{2}=$ Coeficiente de determinación, $* *=$ Altamente significativo $(\mathrm{P} \leq 0,01)$.

Tabla 3. Comparación de medias de tratamientos de las características de semilla de las líneas endogámicas y cruzas simple y trilineal de maíz azul bajo estudio (Instituto Politécnico Nacional, México, 2018).

\begin{tabular}{cccccccc}
\hline Tratamiento & P100 $(\mathrm{g})$ & VR $\left(\mathrm{mm}^{3}\right)$ & LG $(\mathrm{mm})$ & AG $(\mathrm{mm})$ & EG $(\mathrm{mm})$ & VOL $\left(\mathrm{mm}^{3}\right)$ & DR \\
\hline 1 & $22,1 \mathrm{~b}$ & $20,8 \mathrm{~b}$ & $11,6 \mathrm{bc}$ & $7,2 \mathrm{~b}$ & $4,4 \mathrm{~b}$ & $367,3 \mathrm{c}$ & $1,1 \mathrm{~b}$ \\
2 & $21,7 \mathrm{~b}$ & $20,5 \mathrm{~b}$ & $10,9 \mathrm{c}$ & $6,3 \mathrm{c}$ & $5,1 \mathrm{a}$ & $353,2 \mathrm{c}$ & $1,1 \mathrm{~b}$ \\
3 & $16,7 \mathrm{c}$ & $12,2 \mathrm{c}$ & $9,8 \mathrm{~d}$ & $6,6 \mathrm{c}$ & $4,5 \mathrm{~b}$ & $290,2 \mathrm{~d}$ & $1,4 \mathrm{a}$ \\
4 & $31,4 \mathrm{a}$ & $23,0 \mathrm{a}$ & $12,1 \mathrm{ab}$ & $7,5 \mathrm{ab}$ & $4,8 \mathrm{ab}$ & $433,8 \mathrm{~b}$ & $1,4 \mathrm{a}$ \\
5 & $30,3 \mathrm{a}$ & $25,0 \mathrm{a}$ & $12,4 \mathrm{a}$ & $7,9 \mathrm{a}$ & $5,0 \mathrm{a}$ & $494,0 \mathrm{a}$ & $1,2 \mathrm{ab}$ \\
DSH & 2,3 & 2,1 & 0,9 & 0,5 & 0,4 & 55,7 & 0,2 \\
\hline
\end{tabular}

En columnas, letras distintas indicaron tratamientos diferentes (Tukey, $\mathrm{P} \leq 0,05$ ). 
atribuir a efectos heteróticos (Pfeiffer y McClafferty, 2007). Comparando las líneas entre sí, CH1 y CH2 fueron mayores que $\mathrm{CH} 3$ en todas las evaluaciones, excepto en DR en la que esta alcanzó el valor mayor $(\mathrm{P} \leq 0,05)$, así que fue la más compacta junto con $\mathrm{CH} 4$, la cruza simple (Tabla 3).

En promedio de genotipos, la luminosidad ( $\left.L^{*}\right)$ fue 28,3 (Tabla 4), por lo que el color de las semillas de todos ellos fue oscuro y las líneas, por sus valores significativamente más altos, fueron más luminosas que las cruzas.

Los valores de $\mathrm{a}^{*}$ (positivos y reducidos) oscilaron en el morado; $\mathrm{CH} 1$ y $\mathrm{CH} 5$ tuvieron los datos mayores $(\mathrm{P} \leq 0,05)$ ( 3,1 y 3,6 , respectivamente), por eso sus semillas fueron menos moradas (tendieron al rojo). En b*, con valores positivos y cercanos a cero, las líneas se ubicaron en el morado, sobre todo $\mathrm{CH} 2$ y $\mathrm{CH} 3$ que mostraron los valores menores $(\mathrm{P} \leq 0,05)$; mientras que las cruzas, con valores negativos, presentaron un color más azul.

Los valores de c (croma o pureza del color) indicaron una gradación que va del azul grisáceo de $\mathrm{CH} 2$ y CH3, cuyos datos (1,0 y 0,9 , respectivamente) fueron los menores $(\mathrm{P} \leq 0,05)$, al azul más puro de $\mathrm{CH} 1, \mathrm{CH} 4$ y $\mathrm{CH} 5$, cuyo promedio fue de 3,6.

La saturación de color (h) fue menor $(\mathrm{P} \leq 0,05)$ en CH1 $(14,8)$ y correspondió al morado - rojizo, CH2 $(161,5)$ y CH3 $(113,9)$ asumieron una saturación verde - azul y las cruzas $(\mathrm{CH} 4,292,5$ y $\mathrm{CH} 5,332,1)$ mostraron un color azul significativamente $(\mathrm{P} \leq$ $0,05)$ más saturado (intenso).

Las cruzas expresaron contenido menor $(\mathrm{P} \leq$ 0,05) de AT que las líneas, en las cuales CH1 (774,7 $\mu \mathrm{g} \mathrm{Pel/g)} \mathrm{destacó} \mathrm{significativamente} \mathrm{(Tabla} \mathrm{4).} \mathrm{En}$ la evaluación visual del maíz azul se considera que semillas de color azul oscuro e intenso poseen mayor proporción de antocianinas y son más apreciadas. Con base en los datos presentados aquí, este argumento es válido respecto a que el color azul intenso del grano le confiere un fenotipo exótico y apetecible, pero no en cuanto a contenido del pigmento.

Por otra parte, las AT se correlacionaron (Tabla 5) alta y significativamente con las coordenadas del color $\mathrm{h}\left(-70 \% \%^{* *}\right)$ y $\mathrm{b}^{*}\left(53 \% \%^{* *}\right)$. Estos resultados confirmaron, respecto a h, que la cuantía de antocianinas se redujo cuando el color azul se saturó (fue más intenso), fenómeno que se presentó en las cruzas simple y trilineal; y, en referencia $\mathrm{a} \mathrm{b}^{*}$, que las antocianinas disminuyeron cuando la coloración de las semillas se alejó del azul, hecho reportado por Salinas et al. (2013).

Las AT se correlacionaron negativamente con P100 ( $-54 * *)$, EG $(-78 * *)$, VOL $(-47 * *)$ y VR $\left(-53^{* *}\right)$, es decir, las semillas con dimensiones menores tuvieron más pigmento. Este fue el caso de las líneas, cuyo tamaño y peso fueron menores que en las cruzas, pero presentaron significativamente más pigmento $(\mathrm{P} \leq 0,05)$ que ellas (Tablas $3 \mathrm{y} 4)$. Así que hubo efecto heterótico para los caracteres de semilla, pero no para el contenido de antocianinas, hecho contrario a lo establecido por Pfeiffer y McClafferty (2007), quienes consideran que los pigmentos responden a la heterosis.

La correlación negativa de las AT con VOL y VR de las semillas podría explicarse por la semilla

Tabla 4. Comparación de medias de tratamientos de los parámetros del color $\left(\mathrm{L}^{*}, \mathrm{a}^{*}, \mathrm{~b}^{*}, \mathrm{c}, \mathrm{h}\right)$ y contenido de antocianinas (AT, $\mu \mathrm{g}$ Pel/g) de las líneas endogámicas y cruzas simple y trilineal de maíz azul bajo estudio (Instituto Politécnico Nacional, México, 2018).

\begin{tabular}{|c|c|c|c|c|c|c|}
\hline Tratamiento & $\mathrm{L}^{*}$ & $a^{*}$ & $\mathrm{~b}^{*}$ & $\mathrm{c}$ & $\mathrm{h}$ & AT \\
\hline \multicolumn{7}{|c|}{ Líneas } \\
\hline 1 & $28,5 \mathrm{ab}$ & $3,1 \mathrm{a}$ & $0,8 \mathrm{a}$ & $3,3 \mathrm{a}$ & $14,8 \mathrm{c}$ & $774,7 \mathrm{a}$ \\
\hline 2 & $31,0 \mathrm{a}$ & $0,2 \mathrm{~b}$ & $0,7 \mathrm{a}$ & $1,0 \mathrm{~b}$ & $161,5 \mathrm{abc}$ & $387,4 \mathrm{~d}$ \\
\hline 3 & 31,9 a & $0,8 \mathrm{~b}$ & $0,3 \mathrm{a}$ & $0,9 \mathrm{~b}$ & $113,9 \mathrm{bc}$ & $600,4 \mathrm{~b}$ \\
\hline \multicolumn{7}{|c|}{ Cruzas } \\
\hline 4 & $24,6 \mathrm{~b}$ & $1,3 \mathrm{~b}$ & $-3,1 \mathrm{~b}$ & $3,51 \mathrm{a}$ & $292,5 \mathrm{ab}$ & $419,5 \mathrm{c}$ \\
\hline 5 & $25,7 \mathrm{~b}$ & $3,6 \mathrm{a}$ & $-1,8 \mathrm{~b}$ & $4,1 \mathrm{a}$ & $332,1 \mathrm{a}$ & $393,7 \mathrm{~d}$ \\
\hline DSH & 4,9 & 1,6 & 1,4 & 1,0 & 206,5 & 7,5 \\
\hline
\end{tabular}

En columnas, letras distintas indicaron tratamientos diferentes (Tukey, $\mathrm{P} \leq 0,05$ ). 
Tabla 5. Coeficientes de correlación lineal (\%) entre las variables evaluadas en las líneas endogámicas y cruzas simple y trilineal de maíz azul (Instituto Politécnico Nacional, México, 2017).

\begin{tabular}{|c|c|c|c|c|c|c|c|c|c|c|c|c|c|}
\hline & P100 & VR & LG & AG & EG & VOL & DR & $\mathrm{AT}$ & $\mathrm{L}^{*}$ & $a^{*}$ & $\mathrm{~b}^{*}$ & $c$ & $\mathrm{~h}$ \\
\hline P100 & 100 & $85^{* *}$ & $85^{* *}$ & $75^{* * *}$ & $43 \mathrm{~ns}$ & $89 * *$ & $21 \mathrm{~ns}$ & $-54 * *$ & $-81 * *$ & $36 \mathrm{~ns}$ & $-82^{* * *}$ & $78^{* *}$ & $63^{* *}$ \\
\hline VR & & 100 & $90 * *$ & $66^{* * *}$ & $44 *$ & $85^{* *}$ & $-32 \mathrm{~ns}$ & $-42 n s$ & $-68 * *$ & $46^{*}$ & $-51^{*}$ & $75^{* *}$ & $48^{*}$ \\
\hline LG & & & 100 & $72 * *$ & $21 \mathrm{~ns}$ & $85^{* *}$ & $-14 \mathrm{~ns}$ & $-30 \mathrm{~ns}$ & $-73 * *$ & $64^{* *}$ & $-53^{*}$ & $87^{* *}$ & $49 *$ \\
\hline $\mathrm{AG}$ & & & & 100 & $16 \mathrm{~ns}$ & $87 * *$ & $14 \mathrm{~ns}$ & $-10 \mathrm{~ns}$ & $-64 * *$ & $68 * *$ & $-68 * *$ & $-87 * *$ & $46^{*}$ \\
\hline EG & & & & & 100 & $54 * *$ & $-04 \mathrm{~ns}$ & $-78 * *$ & $-11 \mathrm{~ns}$ & $-22 n s$ & $-32 \mathrm{~ns}$ & $5 \mathrm{~ns}$ & $43 \mathrm{~ns}$ \\
\hline VOL & & & & & & 100 & $3 \mathrm{~ns}$ & $-47 *$ & $-67 * *$ & $54 * *$ & $-69 * *$ & $82 * *$ & $61 * *$ \\
\hline DR & & & & & & & 100 & $-53^{* *}$ & $-81 * *$ & $36 \mathrm{~ns}$ & $-82 * *$ & $78^{* *}$ & $63^{* *}$ \\
\hline AT & & & & & & & & 100 & $25 \mathrm{~ns}$ & $26 \mathrm{~ns}$ & $53^{* *}$ & $4 \mathrm{~ns}$ & $-70 * *$ \\
\hline $\mathrm{L}^{*}$ & & & & & & & & & 100 & $-37 \mathrm{~ns}$ & $64 * *$ & $-70 * *$ & $-46^{*}$ \\
\hline$a^{*}$ & & & & & & & & & & 100 & $-8 \mathrm{~ns}$ & $77 * *$ & $13 \mathrm{~ns}$ \\
\hline $\mathrm{b}^{*}$ & & & & & & & & & & & 100 & $-55^{* *}$ & $-71 * *$ \\
\hline $\mathrm{c}$ & & & & & & & & & & & & 100 & $35 \mathrm{~ns}$ \\
\hline $\mathrm{h}$ & & & & & & & & & & & & & 100 \\
\hline
\end{tabular}

$*$ Significativo $(\mathrm{P} \leq 0,05), * *=$ Altamente significativo $(\mathrm{P} \leq 0,01), \mathrm{ns}=$ No significativo.

liviana y de densidad baja característica de los maíces azules. La estrategia para contrarrestar esto sería seleccionar líneas derivadas de materiales con endospermo vítreo (Arellano et al., 2013), o bien, elegir como progenitores hembra a las líneas sobresalientes en cantidad de antocianinas como fue CH1 (Cui et al., 2012).

En el caso de los maíces pigmentados, cuyo valor comercial es superior al de los convencionales (Arellano et al., 2013), tal vez sea rentable sembrar líneas endogámicas sobresalientes en acumulación de antocianinas, las cuales se purifiquen y destinen a la industria de alimentos, cosméticos o bebidas (Boo et al., 2012).

Los valores de correlación de P100 con VR, LG, AG y VOL fueron altos y positivos, hecho esperado por tratarse de variables interdependientes entre sí (Gutiérrez et al., 2014). Estos autores destacaron las correlaciones altas y negativas del P100 con L* $(-81 \% * *)$ y b* $(-82 \% * *)$, así como la que obtuvo con $\mathrm{h}(63 \% * *)$, porque evidenciaron que semillas con peso mayor tuvieron menos brillo $\left(\mathrm{L}^{*}\right)$ y su color fue menos azul ( $b^{*}$ ) (tendieron al amarillo) y poco saturado (h); además, presentaron contenido escaso de AT, como se dijo previamente. El desempeño de todas las mediciones de tamaño (LG, AG, EG) y volumen (VR, VOL, DR) de semilla fue similar al descrito para P100.

El brillo de las semillas ( $\left.\mathrm{L}^{*}\right)$ se correlacionó ( $\mathrm{P}$ $\leq 0,01)$ con $\mathrm{b}^{*}$, c y h, en el primer caso de manera positiva y en los otros negativamente. Esto demostró que cuando las semillas tuvieron más brillo $\left(\mathrm{L}^{*}\right)$ su color azul tendió a ser amarillento (b*), más gris (c) y menos saturado (h). Cabe reiterar que $\mathrm{L}^{*}$ no se asoció con las AT, como sí lo hicieron $\mathrm{b}^{*}\left(53^{* *}\right)$ y h $\left(-70^{* *}\right)$, así que las semillas con coloraciones azul - amarillento y con saturación escasa alcanzaron mayor contenido de antocianinas.

Dado que las AT se asociaron con todas las variables de magnitud de semilla de manera análoga, se tomó al P100 como representativa de ellas y fue una de las variables independientes del modelo de regresión múltiple planteado (Tabla 6), junto con las coordenadas L*, b*, c y h, las cuales se eligieron para analizar la determinación del contenido de antocianinas en función de los parámetros sobresalientes del color de la semilla y de su peso. Este modelo de regresión (AT $=\mathrm{P} 100$ $\left.L^{*} b^{*} \mathrm{c} h\right)$ fue significativo $(\mathrm{P} \leq 0,01)$ y confiable por su variación reducida (C.V. $=15,75 \%)$ y su ajuste alto $\left(\mathrm{R}^{2}=80 \%\right)$ al experimento planteado.

Los coeficientes de regresión significativos $(\mathrm{P} \leq$ $0,01)$ del modelo probado fueron de P100 $(-36,05)$, c $(90,93)$ y h $(-0,47)$. Estos datos demostraron que el contenido de AT se redujo al aumentar el peso de las semillas (P100) y también cuando el color de estas fue menos grisáceo (más azul) (c) y el azul tendió a saturarse (h).

Los coeficientes estandarizados de los regresores significativos fueron $-1,35,-0,45$ y 0,84 para $\mathrm{P} 100$, h y c, respectivamente, lo cual denotó que el peso de semillas y la saturación del color azul contribuyeron negativamente (sobre todo P100) a la determinación de la cantidad de antocianinas, 
Tabla 6. Análisis de regresión múltiple considerando como regresores del contenido de antocianinas en las líneas endogámicas y cruzas simple y trilineal de maíz azul bajo estudio, a cuatro coordenadas del color y al peso de semillas (AT $=$ P100 L* b* c h) (Instituto Politécnico Nacional, México, 2018).

\begin{tabular}{|c|c|c|c|c|c|}
\hline Variable & Coeficiente de regresión & Error estándar & & \multicolumn{2}{|c|}{$\begin{array}{c}\text { Coeficiente de regresión } \\
\text { estandarizado }\end{array}$} \\
\hline P100 & $-36,05$ & 8,89 & $* *$ & & \\
\hline $\mathrm{L}^{*}$ & $-13,26$ & 9,06 & ns & & \\
\hline$b^{*}$ & $-22,16$ & 21,65 & ns & & \\
\hline $\mathrm{c}$ & 90,93 & 22,60 & $* *$ & & \\
\hline $\mathrm{h}$ & $-0,47$ & 0,19 & $* *$ & & \\
\hline Intercepto & 1612,63 & & & & \\
\hline Modelo & \multicolumn{2}{|c|}{ Cuadrados medios } & & C.V. $(\%)$ & $\mathrm{R}^{2}(\%)$ \\
\hline $\mathrm{AT}=\mathrm{P} 100 \mathrm{~L}^{*} \mathrm{~b}^{*} \mathrm{ch}$ & $\begin{array}{l}\text { Modelo } \\
\text { Error }\end{array}$ & $\begin{array}{l}73491 \\
6580,09\end{array}$ & $* *$ & 15,75 & 80 \\
\hline
\end{tabular}

** = Altamente significativo $(\mathrm{P} \leq 0,01), \mathrm{ns}=$ No significativo, C.V. $=$ Coeficiente de variación, $\mathrm{R}^{2}=$ Coeficiente de determinación.

pero que la aportación de la pureza del color azul fue positiva y de magnitud considerable $(0,84)$. Por tanto, las antocianinas tuvieron una relación muy estrecha con el color de la semilla del maíz azul (Betrán et al., 2001; Hernández-Uribe et al., 2007; Salinas et al., 2013).

\section{Conclusiones}

Respecto a las cruzas simple y trilineal, las semillas de las líneas parentales mostraron dimensiones menores, su color azul fue más luminoso, de menor pureza (grisáceo) y con saturación escasa (poco vívido), y su contenido de antocianinas resultó mayor. Los valores de correlación y de regresión indicaron que el contenido de antocianinas se asoció negativamente con las dimensiones de las semillas $\mathrm{y}$, positivamente, con el color azul poco saturado y de luminosidad reducida. Las variables con mayor contribución a la cantidad de antocianinas fueron, negativamente, el peso de semilla y la saturación, $\mathrm{y}$, de manera positiva, la pureza del color.

\section{Literatura Citada}

Alvis, A.; Romero, P.; Granados, C.; Torrenegra, M.; PájaroCastro, N.

2017. Evaluación del color, las propiedades texturales y sensoriales de salchicha elaborada con carne de babilla (Caiman crocodilus fuscus). Revista Chilena de Nutrición, 44: 89-94.

Arellano V., J.L.; Rojas M., I.; Gutiérrez H., G.F.

2013. Híbridos y variedades sintéticas de maíz azul para el Altiplano Central de México: potencial agronómico y estabilidad del rendimiento. Revista Mexicana de Ciencias Agrícolas, 4 (7): 999-1011.

Arellano V., J.L.; Rojas M., I.; Gutiérrez H., G.F.

2014. Variedades de maíz azul Chalqueño seleccionadas por múltiples caracteres y estabilidad del rendimiento. Revista Mexicana de Ciencias Agrícolas, 5 (18): 1469-1480.

Betrán, F.J.; Bockholt, A.J.; Rooney, L.W. 2001. Blue corn. In: A.R. Hallauer (ed.). Speciality corns. Iowa State University. Ames, Iowa, USA. pp. 293-337.

Boo, H.O.; Hwang, S.J.; Bae, C.S.; Park, S.H.; Heo, B.G.; Gorinstein, S.

2012. Extraction and characterization of some natural plant pigments. Industrial Crops and Products, 40: 129-135.
CIE(International Commission on Illumination). 2004. Technical report colorimetry. CIE $1582 \mathrm{p}$.

Cui, L.; Gao, R.; Dong, S.; Zhang, J.; Liu, P.; Zhang, H.; Meng, J.; Shi, D.

2012. Effects of ear shading on the anthocyanin contents and quality of kernels in various genotypes of maize. Australian Journal of Crop Science, 6 (4): 704-710.

Galicia, L.; Miranda, A.; Gutiérrez, M.G.; Custodio, O.; Rosales,

A.; Ruiz, N.; Surles, R.; Palacios R., N.

2012. Laboratorio de calidad nutricional de maíz y análisis de tejido vegetal. Protocolos de laboratorio. Centro Internacional de Mejoramiento de Maíz y Trigo. México, D.F. 50 p.

Gutiérrez H., G.F.; Arellano V., J.L.; Vázquez R., J. M.; García R., E.; Vázquez L., P.; Flores G., E.

2014. Formación de híbridos de maíz con calidad proteica: lisina, triptófano e índice de calidad. Revista de la Facultad de Agronomía Universidad del Zulia, 31: 171-189.

Hernández-Uribe, J.P.; Agama-Acevedo, E.; Islas-Hernández, J.J.; Tovar, J.; Bello-Pérez, L.A.

2007. Chemical composition and in vitro starch digestibility of pigmented corn tortilla. Journal Science of Food Agriculture, 87: 2482-2487. 
IBPGR (International Board for Plant Genetic Resources). 1991. Descriptores para maíz. Centro Internacional de Mejoramiento de Maízy Trigo (CIMMYT). Roma, Italia. 88 p. Kato Y., T.Á.; Mapes S., C.; Mera O., L.M.; Serratos H., J.A.; Bye B., R.A.

2009. Diversificación y distribución reciente del maíz en México. Origen y diversificación del maíz. México, D.F. 116 p.

Lee, E.A.; Tollenaar, M.

2007. Physiological basis of successful breeding strategies for maize grain yield. Crop Science, 47: 202-215.

López-Martínez, L.X.; Oliart-Ros, R.M.; Valerio-Alfaro, G.;

Lee, C.H.; Parkin, K.L.; García, S.H.

2009. Antioxidant activity, phenolic compounds and anthocyanins content of eighteen strains of Mexican maize. LWT-Food Science and Technology, 42: 1187-1192.

McGuire, R.G.

1992. Reporting of objective color measurements. HortScience, 27: 1254-1255.

Mendoza-Mendoza, C.G.; Mendoza-Castillo, M.C.; DelgadoAlvarado, A.; Castillo-González, F.; Kato-Yamakake, T.A.; Cruz-Izquierdo, $\mathrm{S}$.

2017. Antocianinas totales y parámetros de color en líneas de maíz morado. Revista Fitotecnia Mexicana, 40(4): 471-479.

Yang, Z.; Chen, Z.; Yuan, S.; Zhai, W.; Piao, X.; Piao, X.

2009. Extraction and identification of anthocyanin from purple corn (Zea mays L.). International Journal of Food Science and Technology, 44: 2485-2492.
Pfeiffer, W.H.; McClafferty, B.

2007. HarvestPlus: breeding crops for better nutrition. Crop Science, 47: S88-S105.

Salinas M.Y.; Soto H.M.; Martínez B.F.; González H.V.A.; Ortega P.R.

1999. Análisis de antocianinas en maíces de grano azul y rojo provenientes de cuatro razas. Revista Fitotecnia Mexicana, 22: 161-174.

Salinas M., Y.; García S., C.; Coutiño E., B.; Vidal M., V.A. 2013. Variabilidad en contenido y tipos de antocianinas en granos de color azul/ morado de poblaciones mexicanas de maíz. Revista Fitotecnia Mexicana, 36: 285-294.

SAS (Statistical Analysis System).

2002. SAS Institute. Inc. Cary, N.C., USA. Ver. 9.2.

SNICS (Sistema Nacional de Inspección y Certificación de Semillas).

2014. Guía técnica para la descripción varietal de maíz (Zea mays L.). Secretaría de Agricultura, Ganadería, Pesca y Alimentación. Ciudad de México, México. 39 p.

Soto-Vaca, A.; Gutiérrez, A.; Losso, J.N.; Xu, Z.M.; Finley, J.W. 2012. Evolution of phenolic compounds from color and flavor problems to health benefits. Journal of Agricultural Food Chemical, 60: 6658-6677.

Wang, Y.; Zeng, M.

2009. Studies on Zhuo-Zi N ${ }^{\circ}$, a purple hybrid in maize (Zea mays L.). Maize Genetics Cooperation Newsletter, 83: 2-3. 